\title{
30-Day Morbidity and Mortality Rates in Elderly Subjects Following Surgical Tracheostomy
}

\author{
Sharon Ovnat Tamir MD, Nadia Khalaily, Sharon Einav MD MSc, Shay Shemesh MD, \\ Ofer Gluck MD, and Tal Marom MD
}

\begin{abstract}
BACKGROUND: Tracheostomy is considered to be effective in the respiratory support of mechanically ventilated patients. We studied a single-center experience of surgical tracheostomy in mechanically ventilated patients to describe the demographics, risk factors, and outcomes of early $(\leq \mathbf{1 4} \mathbf{d}$ after ventilation) versus late surgical tracheostomy ( $\geq 15 \mathrm{~d}$ after ventilation). METHODS: In this retrospective study, we collected demographic data, medical history, timing of surgical tracheostomy in relation to ventilation day, blood test results, preoperative surgical assessment (subjective impression of neck length, difficulty in neck extension, presence of a goiter), intraoperative complications (bleeding $>100 \mathrm{~mL}$, difficulties in cannula insertion), and postoperative morbidities (bleeding, wound infection, fever, inadvertent de-cannulation, and 30-d postoperative mortality rate) of subjects who underwent surgical tracheostomy in a secondary medical center during 2010-2015. Morbidity and mortality rates were compared between the early versus late surgical tracheostomy groups. RESULTS: Three hundred eleven subjects underwent surgical tracheostomy and met the eligibility criteria. Most of subjects were elderly, with a mean age of 82 y (range 62.5-88 y). There were $22(7 \%)$ subjects in the early surgical tracheostomy group and $289(93 \%)$ subjects in the late surgical tracheostomy group. Late surgical tracheostomy subjects were significantly older compared to early surgical tracheostomy subjects (median age 82 y vs 74 y, $P=.001$ ). With regard to intraoperative complications, no appreciable differences were observed between the groups. Timing of surgical tracheostomy was not associated with greater morbidity rates, nor was timing associated with higher postoperative complication rates. Those who survived $30 \mathrm{~d}$ were younger than those who died (median 81 vs 83 years, hazard ratio $=1.03$ ). CONCLUSION: In elderly subjects, late surgical tracheostomy was not associated with increased 30-d morbidity or mortality rates. Comorbid conditions and subject age had a greater association with 30-d mortality rate than surgical tracheostomy timing. Key words: tracheostomy; elderly; mortality; morbidity; complication. [Respir Care 2018;63(8):1009-1015. (C) 2018 Daedalus Enterprises]
\end{abstract}

\section{Introduction}

Hospitalized critically ill patients often require prolonged mechanical ventilation via an endotracheal tube. This procedure is associated with complications, such as ventilator-associated pneumonia or subglottic and laryngeal stenosis. $^{1,2}$ Therefore, when weaning attempts from me-

\footnotetext{
Drs Tamir, Shemesh, Gluck, and Marom are affiliated with the Department of Otolaryngology-Head and Neck Surgery, Assuta Ashdod University Hospital, Ben Gurion University Faculty of Health Sciences, Ashdod, Israel. Ms Khalaily is affiliated with the Faculty of Medicine, Tel Aviv University Sackler School of Medicine, Tel Aviv, Israel. Dr Einav is affiliated with the Surgical Intensive Care Unit, Shaare Zedek Medical Center, Hebrew University-Hadassah Faculty of Medicine, Jerusalem, Israel.
}

The authors have disclosed no conflicts of interest. chanical ventilation are unsuccessful, or when recovery to spontaneous breathing is not expected within a few days, many ventilated patients are scheduled for tracheostomy. Tracheostomy can be performed either as an open procedure (ie, surgical tracheostomy), which is predominantly performed by otolaryngologists, or as a closed procedure (ie, percutaneous dilatational tracheostomy) if there are no contraindications, which is mainly performed by internists, anesthesiologists, or general surgeons. ${ }^{3}$

\footnotetext{
Correspondence: Tal Marom MD, Department of Otolaryngology-Head and Neck Surgery, Assuta Ashdod University Hospital, Faculty of Health Sciences, Ben Gurion University of the Negev, 7 Ha'refua Street, 77476 Ashdod, Israel. E-mail: talmarom73@gmail.com.
}

DOI: $10.4187 /$ respcare. 06002 
Studies showed that up to $34 \%$ of patients who require mechanical ventilation for $>48 \mathrm{~h}$ undergo tracheostomy for prolonged mechanical ventilation. ${ }^{4}$ Advantages of ventilation via a tracheostomy tube include greater airway security, improved patient comfort, easier and more efficient secretion removal, and facilitation of the transfer of such patients to rehabilitative institutions. ${ }^{5}$ However, tracheostomy may be associated with complications, such as inadvertent decannulation, minor/major bleeding, wound infection, subcutaneous emphysema, pneumothorax/mediastinum, tracheal stenosis, tracheoesophageal fistula formation, tracheomalacia, and occasionally death (intra/postoperatively). ${ }^{6}$ Controversy exists regarding the ideal timing for performing surgical tracheostomy in patients undergoing mechanical ventilation.

This study was designed to examine 30-d morbidity and mortality rates after surgical tracheostomy of all subjects operated on in a single institution and to compare the 30-d morbidity and mortality rates between subjects undergoing early surgical tracheostomy ( $\leq 14 \mathrm{~d}$ from ventilation) and late surgical tracheostomy ( $\geq 15 \mathrm{~d}$ after ventilation). We hypothesized that late surgical tracheostomy would be associated with more complications than early surgical tracheostomy because these patients are ventilated for longer periods, are destabilized by the longer hospitalization, and are more likely to have comorbidities such as concomitant infection or anemia.

\section{Methods}

The study was approved by the local institutional review board (protocol number: WOMC-16-0059).

\section{Clinical Setting}

Our secondary medical care center serves about 300,000 adults. Local law stipulates that informed consent is required for tracheostomy, and family members or caregivers of candidates should obtain legal guardianship from the local family court. This process may take several business days. The decision to perform tracheostomy is usually made $7-10 \mathrm{~d}$ after mechanical ventilation, and after at least 1 trial of extubation has been tried. In some cases, the decision is made even earlier if the chances for extubation are estimated to be very low. Surgical tracheostomies are performed by different otolaryngologists in a standard technique: incision of the second or third tracheal ring (with or without removal of cartilage) and an insertion of a cannula.

\section{Inclusion Criteria}

We retrospectively identified all adult patients (age $\geq 18 \mathrm{y}$ ) who were dependent on mechanical ventilation and underwent surgical tracheostomy in our hospital between January 1, 2010, to December 31, 2015, using International Classification of Diseases-9 discharge codes 31.1 (temporary trache-

\section{QUICK LOOK}

\section{Current knowledge}

Tracheostomy is a common procedure in mechanically ventilated patients who cannot be weaned from the ventilator. Despite advantages for better secretion removal and minimizing the risk for subglottic stenosis, surgical (open) tracheostomy bears potential risks for postoperative complications, including death during or shortly after surgery, especially in older critically ill patients.

\section{What this paper contributes to our knowledge}

In subjects who underwent surgical tracheostomy, we observed a large proportion of older subjects (mean age $82 \mathrm{y}$ ). In these subjects, late surgical tracheostomy ( $\geq 15 \mathrm{~d}$ after ventilation) was not associated with increased 30-d postoperative morbidity or mortality rates compared to subjects who were operated on earlier $(\leq 14 \mathrm{~d}$ from ventilation). Comorbidities such as anemia or COPD and age at surgery had a greater association with 30-d mortality than the timing of tracheostomy.

ostomy) or 31.29 (other permanent tracheostomy, excluding laryngectomy). The medical charts of all eligible adults were reviewed. Patients with previous tracheostomy, who underwent percutaneous dilatational tracheostomy, or who underwent surgical tracheostomy in a trauma setting were excluded, as were patients who underwent tracheostomy in an emergency airway scenario. We could not accurately track the precise indications for mechanical ventilation for each enrolled subject because many had multiple comorbidities, some were receiving mechanical ventilation upon arrival to the hospital, and others failed other noninvasive respiratory support modalities. We included subjects who met eligibility criteria and for whom all data were collected. There were 5 patients for whom all information could not be retrieved or reviewed and were therefore excluded from our analysis.

\section{Study Definitions}

The day of ventilation was defined as day 0 , regardless of the admission day. Fever was defined as the subject having a body temperature was $>38.2^{\circ} \mathrm{C}$ measured orally. Sepsis was defined as the subject having positive bacterial growth in blood cultures with concomitant fever. Bleeding was defined as any excessive bleeding noted by the surgeon in the operative report. Desaturation was defined as an oxygen saturation level $\geq 93 \%$ for $\geq 1$ min. $^{7} \mathrm{We}$ decided on these cutoffs to accurately identify true postoperative infections. 


\section{Study Groups}

All subjects underwent surgical tracheostomy due to chronic respiratory failure, and in most of them at least 2 weaning attempts had failed before surgery (data not shown). Subjects who underwent surgical tracheostomy $\leq 14 \mathrm{~d}$ after ventilation were categorized as early surgical tracheostomy. When tracheostomy was performed on day 15 or later, subjects were classified as late surgical tracheostomy. A 2-week cutoff was determined because prior literature has suggested that this may be the optimal period for performing surgical tracheostomy. ${ }^{4,8,9}$ Multiple potential circumstances may have influenced the timing of surgical tracheostomy, such as medical (eg, hemodynamic instability, infection), technical (eg, availability of operating rooms and surgical staff), or medico-legal (eg, court delays) issues, but these could not be traced or analyzed due to the retrospective nature of this study.

\section{Comorbidities}

Other diagnoses not related to tracheostomy were recorded from each subject's electronic chart and were grouped by the chronic lung disease that was present (most subjects in this category had COPD); by anticoagulation therapy, if the subject had been on any anticoagulation therapy; by cardiovascular issue, if hypertension, ischemic heart disease, valvulopathies, or vasculopathies status post heart surgery were present; by kidney issue, for any acute or chronic renal failure; by liver, for any acute or chronic hepatitis; by neurological, for any peripheral or central nervous system diseases (eg, stroke); and by diabetes.

\section{Data Collection}

For each subject, data were collected regarding 30-d morbidity and mortality from his or her hospital records. Demographic data (eg, age, sex), relevant medical history, timing of surgical tracheostomy, blood test results prior to surgery (ie, hemoglobin, white blood cell count, partial thromboplastin time, international normalized ratio), preoperative surgical assessment (ie, subjective impression of neck length, difficulty in neck extension, the presence of a goiter), intraoperative complications (eg, hemorrhage $>100 \mathrm{~mL}$, difficulty in cannula insertion), and postoperative complications (eg, bleeding, wound infection, sepsis, inadvertent decannulation, airway stenosis, and 30-d postoperative mortality) were recorded. Because many of our subjects had preoperative pneumonia, we did not collect data on postoperative pneumonia. All data were inserted into a dedicated Microsoft Excel spreadsheet (Microsoft, Redmond, Washington).

\section{Statistical Analysis}

Statistical analysis was performed using SPSS (SPSS version 21.0, IBM, Armonk, New York). We calculated that an overall sample size of 250 subjects would reach $80 \%$ power. In the first stage, descriptive statistics were used. Categorical variables (eg, age, timing of surgery) were described as percentage in the group from which they were derived. Continuous variables (eg, age, timing of surgical tracheostomy, white blood cell count, and hemoglobin level) were evaluated for normal distribution using histograms and Q_Q plots. Normally distributed continuous variables were described with their mean \pm SD. Nonnormally distributed variables were described with their median and interquartile ranges (IQRs).

In the second stage, a comparative analysis was performed to determine whether there was an association between study variables (eg, limited neck extension, goiter) using chi-square or Fisher exact tests for categorical variables, and $t$ tests and Mann-Whitney tests for continuous variables. All statistical tests were 2-tailed, and the associations were considered significant when $P \leq .05$.

In the third stage, survival was described with the Kaplan-Meier technique, and survival of subjects in the early surgical tracheostomy group was compared to survival of subjects in the late surgical tracheostomy groups using the $\log$ rank test. Finally, univariate and multivariate Cox proportional hazard models were used to determine the variables associated with mortality after surgical tracheostomy over time. Age, gender, and all variables with a $P<.05$ in the univariate analysis were included in the multivariate analysis to find a possible association between these variables and mortality. The resultant hazard ratios with their 95\% CIs were presented.

\section{Results}

During the study period, 311 subjects underwent surgical tracheostomy and met the eligibility criteria. We classified $22(7 \%)$ subjects in the early surgical tracheostomy group and $289(93 \%)$ subjects in the late surgical tracheostomy group. Among the 22 subjects from the early surgical tracheostomy group, 64\% were males [median age, 74 y (IQR 62.580.75)]; among the 289 subjects from the late surgical tracheostomy group $42 \%$ were males [median age, 82 y (IQR 74-88)]. Thus, our study population can be considered as being mostly composed of older subjects.

Subject Characteristics. Table 1 compares the characteristics of the 2 study groups. Of 311 subjects, 262 (84\%) were $\geq 65 \mathrm{y}$ old. Males were slightly more frequent in the early tracheostomy group $(P=.051)$. Late surgical tracheostomy subjects were significantly older than early surgical tracheostomy subjects (median age 82 y vs 74 y, IQR 
Table 1. Demographics, Comorbidities, and Laboratory Tests Before Surgery

\begin{tabular}{|c|c|c|c|c|}
\hline & $\begin{array}{c}\text { Total } \\
(N=311)\end{array}$ & $\begin{array}{l}\text { Early Tracheostomy } \\
\qquad(n=22)\end{array}$ & $\begin{array}{l}\text { Late Tracheostomy } \\
\qquad(n=289)\end{array}$ & $P$ \\
\hline Day of surgery (IQR)* & $31(23-43)$ & $8(1-11)$ & $33(25-44)$ & \\
\hline Male, $n(\%)$ & $135(43)$ & $14(64)$ & $121(42)$ & .051 \\
\hline Median age, y (IQR) & $82(73-87)$ & $74(62.5-80.75)$ & $82(74-88)$ & .001 \\
\hline \multicolumn{5}{|l|}{ Comorbidities } \\
\hline Chronic lung disease, $n(\%)$ & $59(19)$ & $6(27)$ & $53(18)$ & .39 \\
\hline Anti-coagulant therapy, $n(\%)$ & $154(50)$ & $10(46)$ & $144(50)$ & .69 \\
\hline Cardiovascular diseases, $n(\%)$ & $178(57)$ & $13(59)$ & $165(57)$ & .91 \\
\hline Kidney diseases, $n(\%)$ & $30(10)$ & $6(27)$ & $24(8)$ & .02 \\
\hline Liver diseases, $n(\%)$ & $19(6)$ & $2(9)$ & $17(6)$ & .24 \\
\hline Neurological diseases, $n(\%)$ & $153(49)$ & $12(55)$ & $141(49)$ & .88 \\
\hline Diabetes, $n(\%)$ & $102(33)$ & $10(45)$ & $92(32)$ & .09 \\
\hline \multicolumn{5}{|l|}{ Laboratory tests before surgery } \\
\hline White blood cell count, $\times 10^{3} / \mu \mathrm{L}(\mathrm{IQR})$ & $11.3(8.9-14.3)$ & $11.2(8.8-16)$ & $11.3(9-14.3)$ & .68 \\
\hline Hemoglobin, g/dL (IQR) & $9.5(8.7-10.7)$ & $11.7(9.1-13.5)$ & $9.4(8.6-10.6)$ & .003 \\
\hline Partial thromboplastin time, s (IQR) & $28.2(25.9-30.8)$ & $27.65(25.4-30)$ & $28.2(26-30.8)$ & .27 \\
\hline International normalized ratio (IQR) & $1.06(1.0-1.1)$ & $1.05(1.0-1.1)$ & $1.06(1.2-1.1)$ & .21 \\
\hline $\begin{array}{l}\text { Data is shown as median (IQR), unless otherwise specifiec } \\
\text { * Day of ventilation was considered day } 0 . \\
\mathrm{IQR}=\text { interquartile range }(25-75 \%)\end{array}$ & & & & \\
\hline
\end{tabular}

Table 2. Surgical Findings and Intraoperative Complications

\begin{tabular}{|c|c|c|c|c|}
\hline & $\begin{array}{c}\text { Total } \\
(N=311)\end{array}$ & $\begin{array}{l}\text { Early Tracheostomy } \\
\qquad(n=22)\end{array}$ & $\begin{array}{l}\text { Late Tracheostomy } \\
\qquad(n=289)\end{array}$ & $P$ \\
\hline Limited neck extension, $n(\%)$ & $26(8)$ & $2(9)$ & $24(8)$ & .70 \\
\hline Goiter, $n(\%)$ & $94(30)$ & $6(27)$ & $88(30)$ & .75 \\
\hline Short neck, $n(\%)$ & $8(3)$ & $0(0)$ & $8(3)$ & $>.99$ \\
\hline \multicolumn{5}{|l|}{ Intraoperative complications } \\
\hline Bleeding, $n(\%)$ & $80(26)$ & $2(9)$ & $78(27)$ & .064 \\
\hline Multiple cannula insertions, $n(\%)$ & $18(6)$ & $4(18)$ & $14(5)$ & .18 \\
\hline Pneumothorax, $n(\%)$ & $3(1)$ & $1(5)$ & $2(1)$ & .20 \\
\hline
\end{tabular}

$74-88$ vs $62.5-80.75, P=.001$, respectively) and were more anemic before surgery $(9.4 \mathrm{~g} / \mathrm{dL}$ vs $11.7 \mathrm{~g} / \mathrm{dL}$, respectively, $P=.003$ ). The groups did not significantly differ in other comorbidities, such as chronic lung disease, leukocytosis, anticoagulation therapy, or abnormal coagulation studies. About half of the subjects in each tracheostomy group were on full anticoagulation, while the rest received this therapy for prophylaxis (data not shown). Overall, there were no overt differences in the comorbidities of the study groups, except for renal diseases, which were statistically significantly more common in the early tracheostomy group compared with the late tracheostomy group (27\% versus $8 \%, P=.02)$.

Intraoperative Findings and Complications. Bleeding was the most common intraoperative complication, reported mostly as mild and easily controlled, with a rate of $26 \%$. Table 2 compares surgical findings and intraoperative com- plications of both study groups. Of note, bleeding was more common in the late tracheostomy group, but not statistically significant $(P=.064)$. No other appreciable differences were observed between the groups. There were no postoperative sepsis cases.

30-Day Complications. Fever was the most common postoperative complication (42\%). Table 3 shows the details of postoperative complications as documented within the 30-d period following surgical tracheostomy in each group. There were no cases with postoperative airway stenosis. The timing of surgical tracheostomy was not associated with either greater morbidity rates or higher postoperative complication rates.

30-Day Mortality. Overall 30-d postoperative mortality was high. The 30 -d postoperative mortality rates did not differ between the early and late surgical tracheostomy 
Table 3. Postoperative Complications of Surgical Tracheostomy

\begin{tabular}{|c|c|c|c|c|}
\hline Complication & $\begin{array}{c}\text { Total } \\
(N=311)\end{array}$ & $\begin{array}{l}\text { Early Tracheostomy } \\
\qquad(n=22)\end{array}$ & $\begin{array}{l}\text { Late Tracheostomy } \\
\qquad(n=289)\end{array}$ & $P$ \\
\hline Bleeding, $n(\%)$ & $24(8)$ & $2(9)$ & $22(8)$ & .68 \\
\hline Wound infection, $n(\%)$ & $11(4)$ & $0(0)$ & $11(4)$ & $>.99$ \\
\hline Fever, $n(\%)$ & $132(42)$ & $9(41)$ & $123(43)$ & .88 \\
\hline Accidental decannulation, $n(\%)$ & $20(6)$ & $3(14)$ & $17(6)$ & .16 \\
\hline Subcutaneous emphysema, $n(\%)$ & $2(1)$ & $1(5)$ & $1(0.3)$ & .14 \\
\hline Tracheo-esophageal fistula, $n(\%)$ & $8(3)$ & $0(0)$ & $8(3)$ & $>.99$ \\
\hline Tracheostomy cannula balloon deflation, $n(\%)$ & $1(0.3)$ & $0(0)$ & $1(0.3)$ & $>.99$ \\
\hline
\end{tabular}

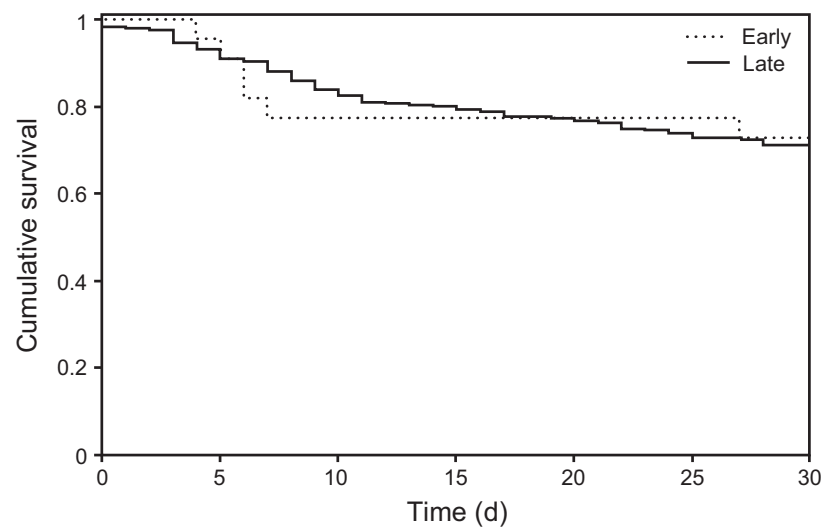

Fig. 1. Kaplan-Meier survival curves for early and late tracheostomy. $P=.92$ at day 30 .

groups (27.3\% and $29.1 \%$ respectively, $P=.92)$. Figure 1 shows the Kaplan-Meier survival curves for both groups. Due to the heterogeneous characterization of this study population and the retrospective nature of this study design, we could not accurately ascertain the precise cause of death because in most records it was noted only as cardiorespiratory failure with no further details.

Variables Affecting Mortality. Despite the relatively old age of the study population, no intraoperative deaths or cerebrovascular accidents occurred. Compared to subjects who died within the 30-d postoperative observation period, those who remained alive tended to be younger (median age 81 vs $83 \mathrm{y}$, hazard ratio $=1.03)($ Table 4$)$. Chronic lung disease was also slightly more frequent in non-survivors $(P=.050)$. Additional independent risk factors associated with higher mortality rates were the presence of chronic lung disease, prolonged international normalized ratio, and limited neck extension (hazard ratios of 1.61, 3.29 , and 1.82 , respectively). No association was observed with the timing of surgical tracheostomy.

\section{Discussion}

In this study, both early and late surgical tracheostomies were associated with similar morbidity and mortality rates.
Older subjects were more likely to undergo late surgical tracheostomy compared to younger subjects. Higher 30-d mortality rate following surgical tracheostomy was associated with older age, chronic lung disease, and variables that most likely reflect a poor general condition.

Patients $>65$ y old make up $24-54 \%$ of ICU admissions in industrialized countries, and patients $\geq 80 \mathrm{y}$ constitute $8-15.3 \%$ of such ICU admissions..$^{7,10,11}$ Elderly patients hospitalized in the ICU have increased morbidity and mortality rates compared to younger patients. ${ }^{11}$ Yet, little is known regarding the specific tracheostomy-related complications in this group of patients. The indication for tracheostomy in elderly patients is a significant concern because it affects not only mortality but also activities of daily living and quality of life after discharge. ${ }^{8}$

Our early and late tracheostomy groups were generally similar in their demographics, comorbidities, and laboratory tests just before surgery, with 3 statistically significant differences. The early group had more males, tended to be younger, and had more history of renal disease. However, their outcomes were not different from the late group. In our opinion, age was the dominant factor that influenced the outcomes measured and reported in this study. Neither gender nor history of renal diseases has previously been recognized as risk factor for increased morbidity or mortality after tracheostomy.

Bleeding has been previously reported to be the most common cause for morbidity and mortality after tracheostomy (open or percutaneous). ${ }^{12,13}$ Therefore, we considered anticoagulation therapy an important variable to measure. In the ICU setting, however, some patients are on full anticoagulation if they have comorbidities that warrant such therapy, such as pulmonary embolism, and some are on partial anticoagulation to prevent thromboembolic events in bedridden patients. About half of our subjects were on this therapy, with a nearly equal difference between full and partial anticoagulation and no differences between the early and the late groups. In this report, anticoagulation therapy was not a considerable cause for increased post-tracheostomy morbidity and mortality rates. 
Table 4. Survivors vs Non-Survivors at $30 \mathrm{~d}$

\begin{tabular}{|c|c|c|c|c|}
\hline & $\begin{array}{l}\text { Survivors } \\
(n=221)\end{array}$ & $\begin{array}{l}\text { Non-Survivors } \\
\quad(n=90)\end{array}$ & $\begin{array}{l}\text { Hazard Ratio } \\
\quad(95 \% \mathrm{CI})\end{array}$ & $P$ \\
\hline Mean age, y (IQR) & $81(70-87)$ & $83(77-89)$ & $1.03(1.01-1.05)$ & .006 \\
\hline Male, $n(\%)$ & $91(41)$ & $44(49)$ & $1.30(0.86-1.96)$ & .22 \\
\hline Late tracheostomy, $n(\%)$ & $205(93)$ & $84(93)$ & $1.05(0.46-2.40)$ & .92 \\
\hline Chronic lung disease, $n(\%)$ & $36(16)$ & $23(26)$ & $1.61(1.00-2.59)$ & .050 \\
\hline Anti-coagulation therapy, $n(\%)$ & $114(52)$ & $40(44)$ & $0.80(0.53-1.21)$ & .28 \\
\hline Hemoglobin level, g/dL (IQR) & $9.6(8.7-10.8)$ & $9.3(8.6-10.4)$ & $0.95(0.84-1.08)$ & .42 \\
\hline White blood cell count, $\times 103 / \mu \mathrm{L}(\mathrm{IQR})$ & $11.2(8.8-14.15)$ & $11.6(9.3-15)$ & $1.01(0.98-1.05)$ & .51 \\
\hline Partial thromboplastin time, $\mathrm{s}$ & $28(25.5-30.6)$ & $28.65(26.4-31.6)$ & $1.02(0.99-1.05)$ & .23 \\
\hline International normalized ratio & $1.06(1.02-1.12)$ & $1.08(1.02-1.17)$ & $3.29(1.14-9.51)$ & .03 \\
\hline Short neck, $n(\%)$ & $5(2)$ & $3(3)$ & $1.37(0.43-4.34)$ & .59 \\
\hline Limited neck extension, $n(\%)$ & $14(6)$ & $12(13)$ & $1.82(0.99-3.34)$ & .053 \\
\hline Goiter, $n(\%)$ & $62(28)$ & $32(36)$ & $1.32(0.86-2.03)$ & .21 \\
\hline Intra-operative bleeding, $n(\%)$ & $54(2)$ & $26(29)$ & $1.19(0.75-1.88)$ & .46 \\
\hline Difficulties in tracheostomy cannula insertion, $n(\%)$ & $5(2)$ & $1(1)$ & $0.56(0.08-3.98)$ & .56 \\
\hline Multiple tracheostomy cannula insertions, $n(\%)$ & $8(4)$ & $4(4)$ & $1.27(0.47-3.47)$ & .64 \\
\hline
\end{tabular}

$\mathrm{IQR}=$ interquartile range $(25-75 \%)$

Halum et al ${ }^{14}$ retrospectively studied 1,175 tracheostomy procedures in 8 hospitals, which were performed by various surgical specialists. They reported much lower rates of bleeding than those observed in our study ( $2.6 \%$ overall vs $26.0 \%$ ). This difference is explained by the variability in the definition of bleeding. They included only severe blood loss as a complication, while we included any excessive bleeding during surgery as mentioned in the surgery logs. Conversely, severe desaturation was the most common intraoperative complication in their study, but this was not the case in our study. This may be due to the relatively high expertise of otolaryngologists in performing surgical tracheostomy, or due to the collection of study from surgical reports and not from anesthesia charts. Otherwise, we report higher complication rates in most categories. A likely cause of these higher rates is the age difference: the average subject age in the our study was 82 y (range 73-87) compared to 57 y (range 15-93) in the Halum study. ${ }^{14}$ We demonstrate that age is a major risk factor: the older the patient, the lower the likelihood survival.

Based on their meta-analysis of 2,037 subjects from 6 studies, Shan et al ${ }^{15}$ concluded that early tracheostomy (performed 3-7 d after intubation) was associated with decreased mortality and reduced ICU length of stay, overall hospital length of stay, and mechanical ventilation duration. We could not extrapolate these findings to our population. Many of the subjects included by Shan et a ${ }^{15}$ were trauma patients with multi-organ injuries. Their population was also significantly younger than ours (mean age $<60 \mathrm{y}$ ). Brook et al ${ }^{16}$ studied 90 medical ICU subjects and found that early tracheostomy (performed $<10 \mathrm{~d}$ after intubation) was associated with shorter hospital length of stay and lower hospital costs, but, similar to our findings, demonstrated no effect on mortality rates.

To our knowledge, our study population is unique in the sense that the subjects were much older than those of other studies reporting on subjects undergoing surgical tracheostomy. Our findings suggest that in the elderly, prolonged endotracheal mechanical ventilation prior to performance of surgical tracheostomy is not associated with higher complication and mortality rates. Our findings are supported by those of Blot et al, ${ }^{17}$ who prospectively randomized 61 subjects to early surgical tracheostomy (on day 4 after intubation) versus 62 controls with continued endotracheal intubation in a multicenter trial. Although their subjects were younger than ours (mean age $55 \mathrm{y}$, range 19-88), they found no relationship between early tracheostomy and 28 -d survival rates (20-24\% mortality rates in both groups). An open multicenter randomized clinical trial performed in the United Kingdom by Young et $\mathrm{al}^{18}$ randomized 909 subjects to either early tracheostomy $(<4 \mathrm{~d}$ after ventilation) or late tracheostomy ( $>10 \mathrm{~d}$ after ventilation, if still indicated). Again, early tracheostomy was not associated with an improvement in the 30-d mortality rate or other important secondary outcomes. In another larger multicenter randomized controlled trial from Italy, Terragni et al ${ }^{19}$ demonstrated that there were no significant differences in intraoperative and postoperative complication rates when tracheostomy was performed early $(<7 \mathrm{~d}$, mean age $61.8 \mathrm{y})$ versus late $(>14 \mathrm{~d}$, mean age $61.3 \mathrm{y}$ ). These findings are in accordance with the systemic review and meta-analysis published by Griffiths et al. ${ }^{20}$ Because the definition of early and late tracheostomy was not uniform in the aforementioned studies (ie, early tracheostomy was defined as being performed $<4,<7$, or $<10 \mathrm{~d}$ after ventila- 
tion), the comparison to our results may be somewhat difficult to interpret.

The main strength of this study is the quality of the data; we performed careful data collection, including comorbidities, which resulted in very little missing data. The fact that all subjects underwent surgical tracheostomy using the same surgical technique reduces the bias that may have been introduced by the use of different tracheostomy techniques. To our knowledge, this is the first study to focus on surgical tracheostomy in an elderly population, which is becoming a substantial group of patients requiring surgical tracheostomy. We acknowledge several limitations to our study. First, our study may have been affected by the need to use ICD codes to identify the subject cohort. Patients with erroneous codes for tracheostomy may have been excluded from the initial database and thus may not have been included in our study population. Second, in this retrospective study we were not able to determine causations for the decision to operate early or late in the hospitalization course after ventilation, only associations. Third, we gathered data from a single center, which may limit the applicability of our findings to other clinical settings. However, as previously noted, our sample size is relatively large compared to other single-center studies, and our findings concur with those of several multicenter trials, which suggests reasonable reliability. ${ }^{16,20,21}$ Fourth, due to the retrospective nature of this study, we could not know if there had been any delays in obtaining informed consent for the procedure by the legal caregivers, or the precise reasons for ICU admission in our study subjects. Therefore, these factors likely contributed to the inequality in the size of the study groups. Finally, the size of the early group was smaller compared to the late group, which could limit the multivariate analysis.

\section{Conclusion}

In an elderly population, performing surgical tracheostomy later than day 14 after ventilation may not be associated with an increase in 30-d morbidity or mortality. Comorbid conditions and the subject's age had a greater association with 30-d mortality than the timing of surgical tracheostomy.

\section{REFERENCES}

1. Neto AS, Barbas CSV, Simonis FD, Artigas-Raventós A, Canet J, Determann RM, et al. Epidemiological characteristics, practice of ventilation, and clinical outcome in patients at risk of acute respiratory distress syndrome in intensive care units from 16 countries (PRoVENT): an international, multicentre, prospective study. Lancet Respir Med 2016;4(11):882-893.

2. Dettmer MR, Damuth E, Zarbiv S, Mitchell JA, Bartock JL, Trzeciak S. Prognostic factors for long-term mortality in critically ill patients treated with prolonged mechanical ventilation: a systematic review. Crit Care Med 2017;45(1):69-74.

3. Vargas M, Sutherasan Y, Antonelli M, Brunetti I, Corcione A, Laffey JG, et al. Tracheostomy procedures in the intensive care unit: an international survey. Crit Care 2015;19:291.
4. Quality of Life After Mechanized Ventilation in the Elderly Study Investigators. 2-month mortality and functional status of critically ill adult patients receiving prolonged mechanical ventilation. Chest 2002; 121(2):549-558.

5. Rumbak MJ, Newton M, Truncale T, Schwartz SW, Adams JW, Hazard PB. A prospective, randomized, study comparing early percutaneous dilational tracheostomy to prolonged translaryngeal intubation (delayed tracheostomy) in critically ill medical patients. Crit Care Med 2004;32(8):1689-1694.

6. Klotz R, Klaiber U, Grummich K, Probst P, Diener MK, Büchler MW, et al. Percutaneous versus surgical strategy for tracheostomy: protocol for a systematic review and meta-analysis of perioperative and postoperative complications. Syst Rev 2015;4:105.

7. Ihra GC, Lehberger J, Hochrieser H, Bauer P, Schmutz R, Metnitz B, et al. Development of demographics and outcome of very old critically ill patients admitted to intensive care units. Intensive Care Med 2012; (4):620-626.

8. Nakarada-Kordic I, Patterson N, Wrapson J, Reay SD. A systematic review of patient and caregiver experiences with a tracheostomy patient. 2018;11(2):175-191.

9. Albert RK, Au DH, Blackford AL, Casaburi R, Cooper JA Jr., Criner GJ, et al. A randomized trial of long-term oxygen for COPD with moderate desaturation. N Engl J Med 2016;375(17):1617-1627.

10. Boumendil A, Woimant M, Quenot JP, Rooryck FX, Makhlouf F, Yordanov Y, et al. Designing and conducting a cluster-randomized trial of ICU admission for the elderly patients: the ICE-CUB 2 study. Ann Intensive Care 2016;6(1):74.

11. Nielsson MS, Christiansen CF, Johansen MB, Rasmussen BS, Tønnesen E, Nørgaard M. Mortality in elderly ICU patients: a cohort study. Acta Anaesthesiol Scand 2014;58(1):19-26.

12. Klemm E, Nowak AK. Tracheotomy-related deaths. Dtsch Arztebl Int 2017;114(16):273-279.

13. Simon M, Metschke M, Braune SA, Püschel K, Kluge S. Death after percutaneous dilatational tracheostomy: a systematic review and analysis of risk factors. Crit Care 2013;17(5):R258.

14. Halum SL, Ting JY, Plowman EK, Belafsky PC, Harbarger CF, Postma GN, et al. A multi-institutional analysis of tracheostomy complications. Laryngoscope 2012;122(1):38-45.

15. Shan L, Hao P, Xu F, Chen YG. Benefits of early tracheostomy: a meta-analysis based on 6 observational studies. Respir Care 2013; 58(11):1856-1862.

16. Brook AD, Sherman G, Malen J, Kollef MH. Early versus late tracheostomy in patients who require prolonged mechanical ventilation. Am J Crit Care 2000;9(5):352-359.

17. Blot F, Similowski T, Trouillet JL, Chardon P, Korach JM, Costa MA, et al. Early tracheostomy versus prolonged endotracheal intubation in unselected severely ill ICU patients. Intensive Care Med 2008;34(10):1779-1787.

18. Young D, Harrison DA, Cuthbertson BH, Rowan K; TracMan Collaborators. Effect of early vs late tracheostomy placement on survival in patients receiving mechanical ventilation: the TracMan randomized trial. JAMA 2013;309(20):2121-2129.

19. Terragni PP, Antonelli M, Fumagalli R, Faggiano C, Berardino M, Pallavicini FB, et al. Early vs late tracheostomy for prevention of pneumonia in mechanically ventilated adult ICU patients: a randomized controlled trial. JAMA 2010;303(15):1483-1489.

20. Griffiths J, Barber VS, Morgan L, Young JD. Systematic review and meta-analysis of studies of the timing of tracheostomy in adult patients undergoing artificial ventilation. BMJ 2005;330(7502):1243.

21. Möller MG, Slaikeu JD, Bonelli P, Davis AT, Hoogeboom JE, Bonnell BW. Early tracheostomy versus late tracheostomy in the surgical intensive care unit. Am J Surg 2005;189(3):293-296. 